\title{
Epstein-Barr Virus-Positive Diffuse Large B-Cell Lymphoma: is it different between Over and Under 50 Years of Age?
}

\author{
Ahmad Monabati ${ }^{1}$,Amir Vahedi ${ }^{2 *}$, Akbar Safaei $^{3}$, Sadat Noori $^{3}$, Maral Mokhtari ${ }^{3}$, \\ Leila Vahedi ${ }^{4}$, Mehdi Zamani ${ }^{3}$
}

\begin{abstract}
Background: Epstein-Barr virus (EBV) positive diffuse large B-cell lymphoma (DLBCL) of the elderly is an entity introduced in the latest WHO classification of lymphoid tumors and defined in patients older than 50 years without prior lymphoma or immunodeficiency. However, recently it has also been seen in patients under 50. There is thus debate as to whether these are separate entities. Materials and Methods: In this retrospective study, we analyzed de novo DLBCL admitted to our institute over a period of two years. Clinical data included age, sex, nodal and extranodal presentation. The results of an immunohistochemistry (IHC) panel were also reviewed. IHC findings were mainly used to sub-classify DLBCL as germinal center vs. non germinal center types. IHC for identification of LMP-1 (latent membrane protein) and in situ hybridization for detection of EBVencoded RNA (EBER) was performed. EBV prevalence, clinical data and IHC findings were compared between patients under and over 50 years of age. Results: Out of 95 DLBCL, $11.6 \%$ were EBV positive $(7.5 \%$ and $14.5 \%$ in the young and old groups). We did not find any significant differences in IHC subclasses and clinical data between EBV positive DLBCL (EBV+DLBCL) of young and old groups. Conclusions: EBV+DLBCL are not exclusive to patients older than 50 years. With regard to clinical data as well as IHC subclasses, no differences were evident between EBV+DLBCL of young and old groups. Our suggestion is to eliminate any cut off age for EBV+DLBCL.
\end{abstract}

Keywords: Epstein barr virus - DLBCL - young age - old age - IHC subclasses

Asian Pac J Cancer Prev, 17 (4), 2285-2289

\section{Introduction}

Lymphoid neoplasm which are derived from B, T or NK cell are among the most common malignancies in the world (Hanif et al., 2009) . Epstein Bar virus (EBV) is a member of the human herpes virus family, which is a DNA virus that was initially detected in a Burkitt's lymphoma (BL) cell culture during 1964 (Dunleavy et al., 2012). Most people get infected with EBV during childhood or adolescence and it can cause infectious mononucleosis in $35 \%$ to $69 \%$ of the cases (Adam et al., 2011) .Epstein Barr virus (EBV) infection is associated with many lymphoproliferative disorders, such as Burkitt lymphoma, Plasmablastic lymphoma, NK/Tcell lymphoma, Angioimmunoblastic T cell lymphoma, Hodgkin lymphoma and immunodeficiency-associated lymphoproliferative disorder (Anagnostopoulos et al., 1992; Kanavaros et al., 1996; Hecht et al., 2000; Barrionuevo et al., 2002, Castillo et al., 2008).

Diffuse large B cell lymphomas (DLBCLs) are the most common type of lymphomas, accounting for 30-
$75 \%$ in different reports (Morton et al., 2006; Akhtar et al., 2009; Kilciksiz et al., 2010; Otrock et al., 2013). The 4th edition of the World Health Organization (WHO) Classification of tumors of hematopoietic and lymphoid tissues included a new entity, "EBV-positive diffuse large B cell lymphoma (DLBCL) of the elderly" (Nakamura et al., 2008). It is defined as an EBV+ DLBCL of patients older than 50 , who had no prior lymphoma or underlying immunosuppression (Nakamura et al., 2008). The incidence rate of EBV in DLBCL patients of Asian or Latin American (8 to $15 \%$ ) is higher than Western countries (less than5\%) (Oyama et al., 2007; Park et al., 2007; Gibson et al., 2009; Morales et al., 2010; Beltran et al., 2011), the highest (14.9\%) is from Peru (Beltran et al., 2011). The EBV incidence is related to environmental and ethnical predisposition and varies in different parts of the world. Most EBV+DLBCL cases have been observed in patients over 60 with median age of 70-79 years (Kuze et al., 2000; Shimoyama et al., 2008; Cohen et al., 2009; Beltran et al., 2011; Dojcinov et al., 2011).

In reports from Asia, patients with $\mathrm{EBV}+\mathrm{DLBCL}$ 
have older age, more advanced stage and more extranodal involvement in comparison with EBV- DLBCL (Park et al., 2007) . Their overall survival rate was worse than EBVDLBCLs (Park et al., 2007; Dojcinov et al., 2011; Ok et al., 2015). Few studies about lymphomas have been done previously in Yazd, center of Iran in a limited number of cases (Binesh et al., 2013). Only few studies have focused on the EBV status in DLBCL of young adults (Cohen et al., 2014; Hong et al., 2015; Ok et al., 2015; Uccini et al., 2015). In this study we investigate the frequency of EBV infection, immunohistochemical subclasses and clinical data in DLBCL of patients younger and older than 50 years of age. Also we compared the phenotype characteristics and clinical parameters in those who were EBV positive in young and old groups. We attempted to answer two questions: 1- Is EBV+ DLBCL, exclusive to older than 50 years? 2- If not, are EBV + DLBCL under 50 phenotypically and clinically different from who are older than 50 ?

\section{Materials and Methods}

In this retrospective study, the medical records of all patients who were diagnosed with DLBCL between 20122014, were retrieved from archives of Hematopathology ward in Shahid Fagihi hospital affiliated to Shiraz University of Medical Sciences. Our archive is one of the largest referral centers for hematopathology consultation in Iran which covers mainly the Southern part of the country. During those years all the diagnosed DLBCL had been made according to the latest WHO classification .Diagnosis were based on morphology and immunohistochemistry (IHC) panel (CD3, CD5, CD20, Pax5, CD10, CD30, BCL2, BCL6, MUM1, Ki67) (Figure 1). Cases with prior diagnosis of lymphoma, immunosuppression, and transplantation or inadequate samples as well as clinical data were excluded in this study. From 120 cases of DLBCL, 25 cases were excluded and 95 enrolled in this study. To investigate EBV frequency, IHC for identification of LMP-1 (latent membrane protein, monoclonal antibodies clones CS. 1-4, DAKO) and in situ hybridization for detection of EBV-encoded RNA (EBER: Zyto Vision Kit) was performed (Figure 1E, 1F). EBER is considered positive, when nuclear staining is seen in more than $20 \%$ of tumor cells (Park et al., 2007). To classify DLBCLs as germinal center (GC) versus non germinal center (N-GC) types, Hans algorithm by the aid of CD10, BCL6 and MUM1 markers was used. Cases with positive CD10 reaction were considered as GC type (Figure 1D). Also cases with negative CD10, positive BCL6 and negative MUM1 were classified as GC type (Hans et al., 2004). Any other profile was regarded as $\mathrm{N}-\mathrm{GC}$. For each patient, EBV status, IHC results (GC vs. Non-GC and CD30 status) and clinical data (age, sex, nodal $v s$. extranodal involvement) were analyzed. This study is the first report about EBV prevalence in youth DLBCL from Iran. The study was approved by the Ethic Committee of Shiraz University of Medical Sciences. All statistical analysis was performed using the SPSS version 20. Comparison of parameters between DLBCL of young and old adults was performed by Chi square test and a $\mathrm{p}<0.05$ was considered significant.

\section{Results}

A total of 95 cases were enrolled in this study. Fifty five of them were older than 50 years and 40 were younger. Mean age was 53.9 for all patients, 37.2 and 66 years for young and old group respectively. Of all 95 cases, 52 were males and 43 were females, $53.6 \%$ had extranodal involvement and $27.3 \%$ were GC type. CD30 positivity was seen in $15.7 \%$ of all. Total numbers of 11 cases (11.6\%) were EBV positive (positive EBER reaction). Among them, 8 (72.7\%) were LMP positive.

In comparison of young and old group, the prevalence of EBV positivity was $7.5 \%$ (3/40) and $14.5 \%(8 / 55)$ respectively $(\mathrm{p}=0.289$, Chi square test).There was no gender predominance (1.12 and $1.29 \mathrm{M} / \mathrm{F}$ ratio). Extranodal involvement were seen in 51 cases $(53.6 \%)$, 18 of them were younger than 50 years and 33 of them were older ( $\mathrm{p}=0.148$, Chi square test). Of total 95 cases, $26(27.3 \%)$ were GC type, 11 belonged to the young and 15 to the old group, without any statistical difference ( $P>0.05$, Chi square test). CD30 positivity were seen in 15 cases $(15.7 \%), 7$ of them were younger than 50 years and 8 were older $(\mathrm{p}=0.697$, Chi square test) (Table 1$)$.

In EBV+ DLBCL, mean age of young and old group were 41 and 66.25 years respectively. We did not find any

Table 1. Clinical and IHC Characteristics in DLBCL Related to EBV Status and age Group

\begin{tabular}{|c|c|c|c|c|c|c|c|}
\hline EBV status & & EBV positive & & & EBV negative & & \\
\hline Age distribution & $\begin{array}{c}\text { Older than } \\
50\end{array}$ & $\begin{array}{c}\text { Younger } \\
\text { than50 }\end{array}$ & total & $\begin{array}{c}\text { Older than } \\
50\end{array}$ & $\begin{array}{c}\text { Younger } \\
\text { than50 }\end{array}$ & total & Total \\
\hline Number(\%) & $8(14.5 \%)$ & $3(7.5 \%)$ & $11(11.6 \%)$ & $47(85.5 \%)$ & $37(92.5 \%)$ & $\begin{array}{c}84 \\
(88.4 \%)\end{array}$ & 9.5 \\
\hline Mean age,years(range) & $\begin{array}{c}66.25 \\
(53-75)\end{array}$ & $41(35-46)$ & $\begin{array}{c}59.36 \\
(35-75)\end{array}$ & $66(51-90)$ & $36.91(12-49)$ & $\begin{array}{c}53.19 \\
(12-90)\end{array}$ & 53.9 \\
\hline Male/Female & 1.66 & 2 & 1.75 & 1.23 & 1.05 & 1.15 & 1.2 \\
\hline Extranodal involvement $(\%)$ & $5(62.5 \%)$ & $2(66.6 \%)$ & $7(63.6 \%)$ & $28(59.5 \%)$ & $16(43.2 \%)$ & $\begin{array}{c}44 \\
(52.3 \%)\end{array}$ & $51(53.6 \%)$ \\
\hline Germinal center type $(\%)$ & $2(25 \%)$ & $1(33.3 \%)$ & $3(27.2 \%)$ & $13(27.6 \%)$ & $10(27 \%)$ & $\begin{array}{c}23 \\
(27.3 \%)\end{array}$ & $26(27.3 \%)$ \\
\hline CD30 positivity(\%) & $2(25 \%)$ & $1(33.3 \%)$ & $3(27.2 \%)$ & $3(27.2 \%)$ & $6(16.2 \%)$ & $\begin{array}{c}12 \\
(14.2 \%)\end{array}$ & $15(15.7 \%)$ \\
\hline LMP positivity $(\%)$ & $5(62.5 \%)$ & $3(100 \%)$ & $8(72.7 \%)$ & - & - & - & $8(72.7 \%)$ \\
\hline
\end{tabular}




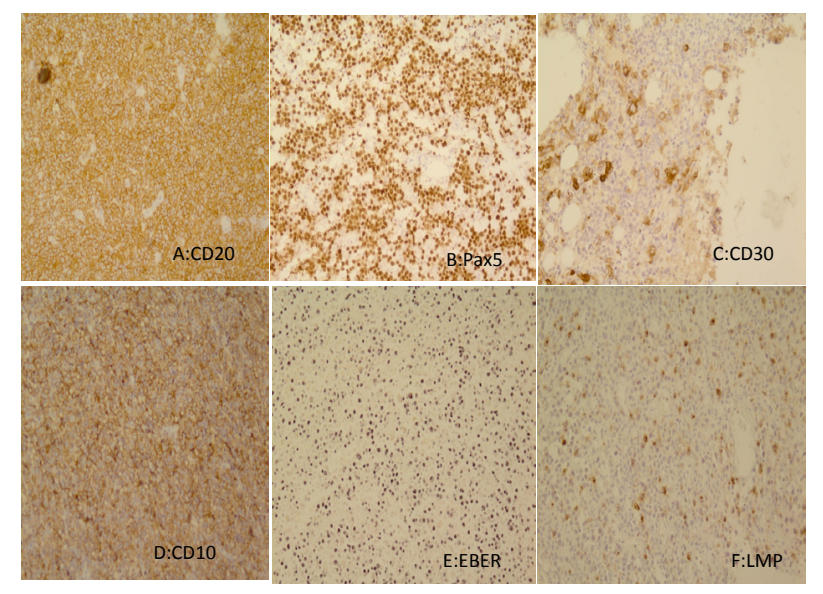

Figure 1. Different immunohistochemical reactions of CD20, pax5, CD30, CD10, EBER and LMP. A) Strong and diffuse positive reaction of CD20 in an EBV+DLBCL of 62 year old man. B) Strong nuclear staining of pax 5 in an EBV+DLBCL of 43 year old man. C) Scattered expression of CD30 in large cells of 57 year old man with EBV+DLBCL. D) Diffuse expression of CD10, indicative of GC type subclasses in a 37 year old woman. E) Diffuse and strong nuclear staining for EBER in the same case as B. F) LMP in a 37 year old man with EBV+DLBCL

significant differences in clinical data, GC type prevalence (33.3\% vs. $25 \%)$ and CD30 positivity (33.3\% vs. 25\%) between EBV+ DLBCL of young and old age patients (Table 1). LMP positive reaction was seen more in young patients $(100 \%$ vs. $62.5 \%)(\mathrm{P}>0.05$, Chi square test).

In comparison between EBV positive and EBV negative DLBCL, the frequency of CD30 expression in $\mathrm{EBV}+\mathrm{DLBCL}$ was higher than EBV - DLBCL (27.2\% vs. $14.2 \%)(\mathrm{p}=0.26 \mathrm{Chi}$ square test $)$. Also extranodal involvement in EBV + DLBCL was higher than negative ones $(63.6 \%$ vs. $52.3 \%)$ (Table 1$)$.

\section{Discussion}

DLBCL comprises of different entities with diverse pathogenesis and clinical behavior. EBV-positive DLBCL of the elderly is a new entity in the current WHO classification. It is seen in patients older than 50 years without prior lymphoma and immunodeficiency (Sabattini et al., 2010). It is associated with more advanced stage and extranodal involvement (Park et al., 2007). Mechanism of lymphomagenesis is related to latent membrane protein-1 (LMP-1) and decreased cytotoxic T-cell effector responses (immunosenescence) (Aw et al., 2007). Ishtiaq et al, stated that frequency of EBV in NHL is $12.7 \%$ and is mostly seen in diffuse large B cell lymphoma (Ishtiaq et al., 2013). There are a few EBV+DLBCL reports in young adults (Beltran et al., 2011; Ao et al., 2014; Cohen et al., 2014; Hong et al., 2015; Uccini et al., 2015). Hong et al., stated that EBV + DLBCL in young adults is a different entity which has distinct pathogenesis and behavior in compare to EBV+DLBCL of the elderly (Hong et al., 2015). Cohen et al., found that this entity is not restricted to elderly patient and suggested to revise the cutoff age in WHO classification (Cohen et al., 2014). Recently OK et al., in a comprehensive gene expression study concluded that EBV+DLBCL in $<50$ years versus $>50$ have similar clinicopathologic, immunophenotypic and genetic features (Ok et al., 2015). Hence, the prevalence of EBV+ DLBCL in our study was $11.6 \%$, which is comparable with the results of Asian and American studies (Kuze et al., 2000; Oyama et al., 2007; Park et al., 2007; Cohen et al., 2014; Hong et al., 2015). In this research, the prevalence of EBV+ DLBCL in cases older than 50 years was higher than younger $(14.5 \%$ vs. $7.5 \%)$, which is close to results of the study from Asia (9.3\% vs. 6.7\%) (Hong et al., 2015), and another study from Peru (15\% vs. 2.2\%) (Shimoyama et al., 2009), on the contrary to a report from Argentina (8\% vs.13\%) (Cohen et al., 2014) . Mean age of EBV+ DLBCL in young and old group (41, 66.25 years) is similar to other reports (Beltran et al., 2011; Cohen et al., 2014; Hong et al., 2015). The reason for increased EBV positivity in DLBCL of old patients may be due to defective immune surveillance for EBV which is associated with immunological deterioration as a result of aging process (Kuze et al., 2000; Shimoyama et al., 2008). Most EBV+ DLBCL had extranodal presentation (63.6\%) like 2 reports from Far East Asia (Oyama et al., 2007; Shimoyama et al., 2009) but in contrast to some reports (Gibson et al., 2009; Quintanilla-Martinez et al., 2009; Hoeller et al., 2010; Hofscheier et al., 2011; Cohen et al., 2014; Uccini et al., 2015). Surprisingly, the prevalence of GC type DLBCL was very low in our cases (27.3\%) and we did not find any significant differences between young and old as well as EBV positive and negative groups. There has been only one study in this geographical region in which Uccini et al. (2015) reported 7 Iraqi children with $\mathrm{EBV}+\mathrm{DLBCL}$, which all of them were Non-GC type. In order to recognize the significance of this difference in incidence of GC vs. Non-GC subtypes, further evaluation is required. We also evaluated the clinical data and IHC findings in EBV+ DLBCL and compare them between young and old group. Clinical data (excluding age) and IHC subclasses were not statistically different between these groups. Our study showed that EBV + DLBCL, is not restricted to older patients, even though the prevalence was higher in this group. Also we did not find any significant differences in IHC subclasses of young and old DLBCL. CD 30 expression was also another issue that we had focused on. CD30 expression was frequently seen in $\mathrm{EBV}+\mathrm{DLBCL}$ in comparison to EBV- DLBCL (27.2\% $v s .14 .2 \%)$ which is similar two other reports $(42 \% v s .14 \%$ and $28 \%$ vs. 6.4\%) (Hu et al., 2013; Lu et al., 2015). Strong CD30 positivity in large mature B cells has been associated with higher frequency of EBV infection in tumor cells (Hu et al., 2013) .Our study showed that even though CD30 reaction is predictor of EBV infection, it is neither specific nor sensitive. Also morphology is not very useful in leading us toward a survey for EBV infection. So it is still a matter of debate which DLBCL should be more assessed by EBER.

In conclusion, EBV+DLBCL are not restricted to old age. Also we did not find any significant difference between $\mathrm{EBV}+\mathrm{DLBCL}$ of young and old age patient. We suggest that in the next revision of WHO classification, age cutoff should be removed. 


\section{Acknowledgements}

All substantial contributions to this study are mentioned in the author's contributions. Authors are employees of the Shiraz and Tabriz University Medical Centre. The authors would like to thank the Research Consulting Center (RCC) of Shiraz University of Medical Sciences in their assistant of editing this article.

\section{References}

Adam P, Bonzheim I, Fend F, et al (2011). Epstein-Barr viruspositive diffuse large B-cell lymphomas of the elderly. $A d v$ Anat Pathol, 18, 349-55.

Akhtar S, Haque S. I. u, Wafa S. M, et al (2009). Malignant lymphoma in Al-Qassim, Saudi Arabia, reclassified according to the WHO classification. Saudi Med J, 30, 677-81.

Anagnostopoulos I, Hummel M, Finn T, et al (1992). Heterogeneous Epstein-Barr virus infection patterns in peripheral T-cell lymphoma of angioimmunoblastic lymphadenopathy type. Blood, 80, 1804-12.

Wang Ao. Q. Y, Tian S. Xu. Y, Huang W, et al (2014). A case of EBV positive diffuse large B-cell lymphoma of the adolescent. Int J Clin Exp Med, 7, 307-11

Silva Aw D. A. B, Palmer D. B (2007). Immunosenescence: emerging challenges for an ageing population. Immunol, 120, 435-46.

Barrionuevo C, Anderson V. M, Zevallos-Giampietri E, et al (2002). Hydroa-like cutaneous T-cell lymphoma: a clinicopathologic and molecular genetic study of 16 pediatric cases from Peru. Appl Immunohistochem Mol Morphol, 10, 7-14.

Beltran B. E, Castillo J. J, Morales D, et al (2011). EBV-positive diffuse large B-cell lymphoma of the elderly: A case series from Peru. Am J Hematol, 86, 663-67.

Beltran B. E, Morales D, Quiñones P, et al (2011). EBV-positive diffuse large b-cell lymphoma in young immunocompetent individuals. Clin Lymphoma Myeloma Leuk, 11, 512-6.

Binesh F, Akhavan A, Behniafard N, et al (2013). Clinicopathologic and survival characteristics of childhood and adolescent non Hodgkin's lymphoma in Yazd, Iran. Asian Pac J Cancer Prev, 15, 1585-8.

Castillo J, Pantanowitz L, Dezube B. J (2008). HIV-associated plasmablastic lymphoma: Lessons learned from 112 published cases. Am J Hematol, 83, 804-9.

Cohen J, Kimura H, Nakamura S, et al (2009). Epstein-Barr virus-associated lymphoproliferative disease in nonimmunocompromised hosts: a status report and summary of an international meeting, 8-9 September 2008. Ann Oncol, 20, 1472-82.

Cohen M, Narbaitz M, Metrebian F, et al (2014). Epstein-Barr virus-positive diffuse large B-cell lymphoma association is not only restricted to elderly patients. Int J Cancer, $\mathbf{1 3 5}$, 2816-24.

Dojcinov S. D, Venkataraman G, Pittaluga S, et al (2011). Agerelated EBV-associated lymphoproliferative disorders in the Western population: a spectrum of reactive lymphoid hyperplasia and lymphoma. Blood, 117, 4726-35.

Dunleavy K, Roschewski M, Wilson W.H(2012). Lymphomatoid granulomatosis and other Epstein-Barr virus associated lymphoproliferative processes. Curr Hematol Malig Rep, 7,208-15.

Gibson S. E, Hsi E. D (2009). Epstein-Barr virus-positive B-cell lymphoma of the elderly at a United States tertiary medical center: an uncommon aggressive lymphoma with a nongerminal center B-cell phenotype. Hum Pathol, 40, 653-61.

Hanif M,Zaidi P, Kamal S, et al (2009). Institution-based cancer incidence in a local population in Pakistan: nine year data analysis. Asian Pac J Cancer Prev, 10, 227-30.

Hans C. P, Weisenburger D. D, Greiner T. C, et al (2004). Confirmation of the molecular classification of diffuse large B-cell lymphoma by immunohistochemistry using a tissue microarray. Blood, 103, 275-82.

Hecht J. L, Aster G.C (2000). Molecular biology of Burkitt's lymphoma. J Clin Oncol, 18, 3707-21.

Hoeller S, Tzankov A, Pileri S.A, et al (2010). Epstein-Barr virus-positive diffuse large B-cell lymphoma in elderly patients is rare in Western populations. Hum Pathol, 41, 352-7.

Hofscheier A, Ponciano A, Bonzheim I, et al (2011). Geographic variation in the prevalence of Epstein-Barr virus-positive diffuse large B-cell lymphoma of the elderly: a comparative analysis of a Mexican and a German population. Mod Pathol, 24, 1046-54.

Hong J, Yoon D, Suh C, et al (2015). EBV-positive diffuse large B-cell lymphoma in young adults: is this a distinct disease entity? Ann Oncol, 26, 548-55.

Hu S, Xu-Monette Z. Y, Tzankov A, et al (2013). MYC/BCL2 protein coexpression contributes to the inferior survival of activated B-cell subtype of diffuse large B-cell lymphoma and demonstrates high-risk gene expression signatures: a report from The International DLBCL Rituximab-CHOP Consortium Program. Blood, 121, 4021-31.

Ishtiaq S, Hassan U, Mushtaq S, et al (2013). Determination of Frequency of Epstein-Barr Virus in Non-Hodgkin Lymphomas Using EBV Latent Membrane Protein 1 (EBVLMP1) Immunohistochemical Staining. Asian Pac J Cancer Prev, 14, 3963-7.

Kanavaros P, Briere J, Emile J,et al (1996). Epstein-Barr virus in T and natural killer (NK) cell non-Hodgkin's lymphomas. Leukemia, 10, 84-87.

Kilciksiz S, Pehlivan M, Yilmaz E, et al (2010). The clinical and pathological features of patients with hematological neoplasms: a study of hospital-based Cancer Registry from western Turkey. J BU ON, 16, 505-10.

Kuze T, Nakamura N, Hashimoto Y, et al (2000). The characteristics of epstein-barr virus (EBV)-positive diffuse large B-cell lymphoma: comparison between EBV+ and EBV-cases in Japanese population. Jpn J Cancer Res, 91 , 1233-40.

Lu TX, Liang JH, Miao Y, et al (2015). Epstein-Barr virus positive diffuse large B-cell lymphoma predict poor outcome, regardless of the age. Sci Rep, 5, 12168.

Morales D, Beltran B, De Mendoza F. H, et al (2010). EpsteinBarr virus as a prognostic factor in de novo nodal diffuse large B-cell lymphoma. Leuk Lymphoma, 51, 66-72.

Morton L. M, Wang S. S, Devesa S. S, et al (2006). Lymphoma incidence patterns by WHO subtype in the United States, 1992-2001. Blood, 107, 265-76.

Nakamura S, Jaffe E, Swerdlow S (2008). EBV positive diffuse large B-cell lymphoma of the elderly. WHO classification of tumours of haematopoietic and lymphoid tissues. Lyon: IARC, 243-44.

Ok C. Y, Ye Q, Li L, et al (2015). Age cutoff for Epstein-Barr virus-positive diffuse large B-cell lymphoma-is it necessary? Oncotarget, 6, 13933-46.

Otrock Z. K, Saab J, Aftimos G, et al (2013). A collaborative nationwide lymphoma study in Lebanon: incidence of various subtypes and analysis of associations with viruses. Pathol Oncol Res, 19, 715-22.

Oyama T, Yamamoto K, Asano N, et al (2007). Age-related EBV- 

associated B-cell lymphoproliferative disorders constitute a distinct clinicopathologic group: a study of 96 patients. Clin Cancer Res, 13, 5124-32.

Park S, Lee J, Ko Y. H, et al (2007). The impact of EpsteinBarr virus status on clinical outcome in diffuse large B-cell lymphoma. Blood, 110, 972-8.

Quintanilla-Martinez L, de Jong D, de Mascarel A, et al (2009). Gray zones around diffuse large B cell lymphoma. Conclusions based on the workshop of the XIV meeting of the European Association for Hematopathology and the Society of Hematopathology in Bordeaux, France. $J$ Hematopathol, 2, 211-36.

Sabattini E, Bacci F, Sagramoso C, et al (2010). WHO classification of tumours of haematopoietic and lymphoid tissues in 2008: an overview. Pathologica, 102, 83-7.

Shimoyama Y, Asano N, Kojima M, et al (2009). Agerelated EBV-associated B-cell lymphoproliferative disorders: Diagnostic approach to a newly recognized clinicopathological entity. Pathol Int, 59, 835-43.

Shimoyama Y, Yamamoto K, Asano N, et al (2008). Age-related Epstein-Barr virus-associated B-cell lymphoproliferative disorders: Special references to lymphomas surrounding this newly recognized clinicopathologic disease. Cancer Sci, 99, 1085-91.

Uccini S, Al-Jadiry M. F, Scarpino S, et al (2015). Epstein-Barr virus-positive diffuse large B-cell lymphoma in children: a disease reminiscent of Epstein-Barr virus-positive diffuse large B-cell lymphoma of the elderly. Hum Pathol, 46, 716-24. 